\title{
Fingerprinting Desmosine-Containing Elastin Peptides
}

\author{
Christoph U. Schräder, ${ }^{1}$ Andrea Heinz, ${ }^{1}$ Petra Majovsky, ${ }^{2}$ Christian E. H. Schmelzer ${ }^{1}$ \\ ${ }^{1}$ Institute of Pharmacy, Faculty of Natural Sciences I, Martin Luther University Halle-Wittenberg, Halle (Saale), Germany \\ ${ }^{2}$ Proteome Analytics Research Group, Leibniz Institute for Plant Biochemistry, Halle (Saale), Germany
}

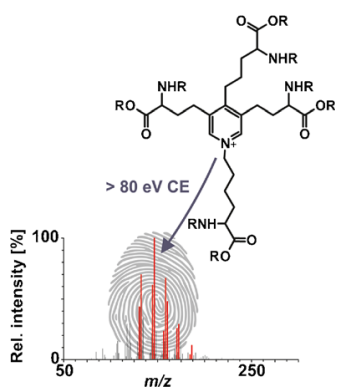

Abstract. Elastin is a vital protein of the extracellular matrix of jawed vertebrates and
provides elasticity to numerous tissues. It is secreted in the form of its soluble
precursor tropoelastin, which is subsequently cross-linked in the course of the elastic
fiber assembly. The process involves the formation of the two tetrafunctional amino
acids desmosine (DES) and isodesmosine (IDES), which are unique to elastin. The
resulting high degree of cross-linking confers remarkable properties, including me-
chanical integrity, insolubility, and long-term stability to the protein. These character-
istics hinder the structural elucidation of mature elastin. However, $\mathrm{MS}^{2}$ data of linear
and cross-linked peptides released by proteolysis can provide indirect insights into
the structure of elastin. In this study, we performed energy-resolved collision-induced dissociation experiments of DES, IDES, their derivatives, and DES-/IDES-containing peptides to determine characteristic product ions. It was found that all investigated compounds yielded the same product ion clusters at elevated collision energies. Elemental composition determination using the exact masses of these ions revealed molecular formulas of the type $\mathrm{C}_{x} \mathrm{H}_{\mathrm{y}} \mathrm{N}$, suggesting that the pyridinium core of DES/IDES remains intact even at relatively high collision energies. The finding of these specific product ions enabled the development of a similarity-based scoring algorithm that was successfully applied on LC-MS/MS data of bovine elastin digests for the identification of DES-/IDES-cross-linked peptides. This approach facilitates the straightforward investigation of native cross-links in elastin.

Keywords: Fragmentation, Low-energy CID, Marker fragments, Mass spectrometry, Native cross-links, Pyridinium

Received: 11 September 2014/Revised: 21 November 2014/Accepted: 17 December 2014/Published online: 21 January 2015

\section{Introduction}

E lastin is an essential protein present in the extracellular matrix of gnathostomata and provides elasticity and resilience to various tissues, including blood vessels, lung, skin, tendon, and cartilage. Elastin content varies highly between $2 \%$ and $5 \%$ of dry weight in the skin and up to $50 \%$ in the aorta (reviewed in [1]). Elastin is secreted as its soluble precursor tropoelastin (TE), whose transcript undergoes extensive alternative splicing. TE consists of alternating hydrophobic and more hydrophilic domains. The latter contain $\mathrm{K}(\mathrm{x}) \mathrm{AAK}$, $\mathrm{KP}(\mathrm{x}) \mathrm{K}$, and KAAAK motifs and are cross-linked upon formation of mature elastin [2]. This cross-linking occurs as a result of oxidative deamination of Lys residues catalyzed by

Electronic supplementary material The online version of this article (doi:10.1007/s13361-014-1075-9) contains supplementary material, which is available to authorized users.

Correspondence to: Christian Schmelzer; e-mail: schmelzer@pharmazie.uni-halle.de lysyl oxidase (LOX) or LOX-like enzymes under formation of highly reactive allysine (k) residues, which further condense - partly with participation of Lys residues - to bi-, tri-, tetra-, and pentafunctional cross-links [3]. These include the tetrafunctional, pyridinium-based amino acids desmosine (DES) and its isomer isodesmosin (IDES) that are exclusively found in elastin [4-6]. It is assumed that about $88 \%$ of all Lys residues in TE take part in cross-linking [7].

Although elastin is resistant to most proteases, some are able to cleave it. Among them are several serine (e.g., cathepsin G), matrixmetallo- (e.g., MMP-12), and cysteine proteases (e.g., cathepsin S) that all share a low site-specificity [8]. Unfortunately, there is no renewal of elastin (with the exception of the uterus) and, thus, its degradation is pathological and associated with severe diseases such as atherosclerosis, lung emphysema, aneurysms in large blood vessels, or solar elastosis in the skin [9-12]. To be able to antagonize such processes, it is of great importance to better understand the structure of elastin, including the formation of its complex cross-linking pattern. In addition to these structural aspects, the screening for DES and IDES 
as potential biomarkers for matrix degradation processes in diseases, such as chronic obstructive pulmonary disease, emphysema [13, 14], and tumor progression [15], has been of great interest over the last three decades. Various methods for the identification and quantification of DES and IDES in body fluids have been reported (reviewed in [16]). DES and IDES are not only released as free amino acids but also to a larger extent in the form of DES-/IDES-containing peptides [13]. To date, however, such cross-linked peptides have not been characterized, especially because of the lack of appropriate methods. Hence, virtually nothing is known about the crosslinking pattern of elastin and its overall structure.

Available software solutions for interpreting MS and tandem MS data of cross-linked peptides mainly deal with chemically introduced and only bifunctional cross-links [17]. However, as mentioned above, elastin also contains a variety of higher-functional cross-links. Fragmentation of such crosslinked peptides is much more complex and has not yet been investigated. Recently, a new software named PolyLinX has been developed by our group for the identification of peptides possessing typical elastin cross-links from MALDI-TOF/TOF MS/MS data of digests of in vitro cross-linked elastin-derived biomaterials [18]. However, sequence elucidation of tetrafunctionally cross-linked peptides from digests of mature elastin remains challenging. This is due to the extremely high number of possible combinations resulting from the many cross-link domains and their high similarity as well as alternative splicing and hydroxylation of Pro residues in mature elastin [19]. The problem is further exacerbated by the fact that proteases with broad specificities have to be used to digest elastin as site-specific enzymes such as the widely used trypsin cannot or not effectively cleave elastin because of the absence of suitable residues or their involvement in cross-linking. To tackle this problem, the differentiation of DES-/IDES-containing peptides from other peptides would be beneficial as it enables their enrichment and thus the acquisition with higher data quality and the performance of additional mass spectrometric or other analytical investigations. A reasonable approach for this detection is the utilization of DES-/IDES-specific fragments.

Dissociation of cross-linked DES-/IDES-containing peptides is characterized by typical backbone fragmentation. As shown in a previous work, these peptides, which have a fixed positive charge at the pyridinium ring, will dissociate especially into larger product ions, of which many contain the cross-link and neutral loss fragments [18]. In this study, we employed nanoESI-QqTOF MS with collision-induced dissociation (CID). The CID in such hybrid instruments is considered to be a low-energy process as collision energies (CEs) below $100 \mathrm{eV}$ are usually used, whereas CID in MALDI-TOF/TOF employs high energies of above $1 \mathrm{keV}$. Another difference lies in the time scale of the dissociations, which is about three orders of magnitude greater in low-energy CID. This, together with a higher efficiency of the energy transfer, results in an overall increased dissociation efficiency [20]. In addition, dissociation can be easily controlled by variation of the $\mathrm{CE}$ and enables energy-resolved studies. Raising the CE often promotes the dissociation of otherwise highly stable bonds and, thus, the release of extra product ions. Among them are immonium ions, which unambiguously indicate the presence of certain residues within a peptide and, therefore, may be useful upon identification of cross-linked peptides, in particular with respect to the elucidation of the cross-linking pattern of elastin. Immonium ions have the general structure $\mathrm{RCH}=\mathrm{NH}_{2}{ }^{+}$ and are formed by a combination of a- and b- or a- and y-type cleavage of peptides during CID [21]. Different studies have investigated the qualitative and quantitative occurrence of these internal ions for the canonical amino acids and their posttranslationally introduced modifications by studying the free amino acids as well as peptides containing specific amino acid residues [22-26]. This raises the question of whether tandem MS of DES and IDES facilitates the release of characteristic fragments which, similar to immonium ions, can be used for the straightforward discovery of these cross-links, especially when DES or IDES is present in larger peptides. Baker and coauthors have described the dissociation of pyridiniumderivatized peptides, which is mainly driven by chargeremote processes [27]. It has been furthermore reported that CID of the doubly charged free amino acids is generally characterized by the loss of small molecules such as $\mathrm{NH}_{3}$ and $\mathrm{CO}$ as well as the break between aliphatic chain and pyridinium nitrogen [28].

The aim of the current study was to gain comprehensive insight into the fragmentation behavior of the most important elastin cross-links, the tetrafunctional amino acids DES and IDES, in an energy-resolved CID study. Focus was set not only on studying the fragmentation behavior of the free cross-linked amino acids but also of several different derivatives of DES and IDES. Moreover, a synthetic DES-/IDES-containing peptide formed by condensation reaction of three identical peptide monomers of the sequence Ac-AAAkAAAKAA was investigated (see Figure 1 and [18]). This model peptide was used to investigate whether the fragmentation behavior of the tetrafunctional amino acid residues changes when they are part of a larger peptide. Based on characteristic fragment ions that were detected upon CID of these DES-/IDES-derived molecules, we aimed to develop an algorithm for the straightforward discovery of DES-/IDES-containing elastin peptides.

\section{Experimental}

\section{Materials}

Porcine pancreatic elastase (PE) as well as DES and IDES standards were obtained from Elastin Products Company (Owensville, MO, USA). Propionic anhydride solution, ammonium bicarbonate, cyanogen bromide, 2-mercaptoethanol, urea, sodium azide, sodium chloride, and trypsin from porcine pancreas were obtained from Sigma Aldrich (Steinheim, Germany). Hydrochloric acid was purchased from Grüssing GmbH (Filsum, Germany) and analytical grade Tris, trifluoroacetic acid (TFA), and formic acid (FA) were obtained 


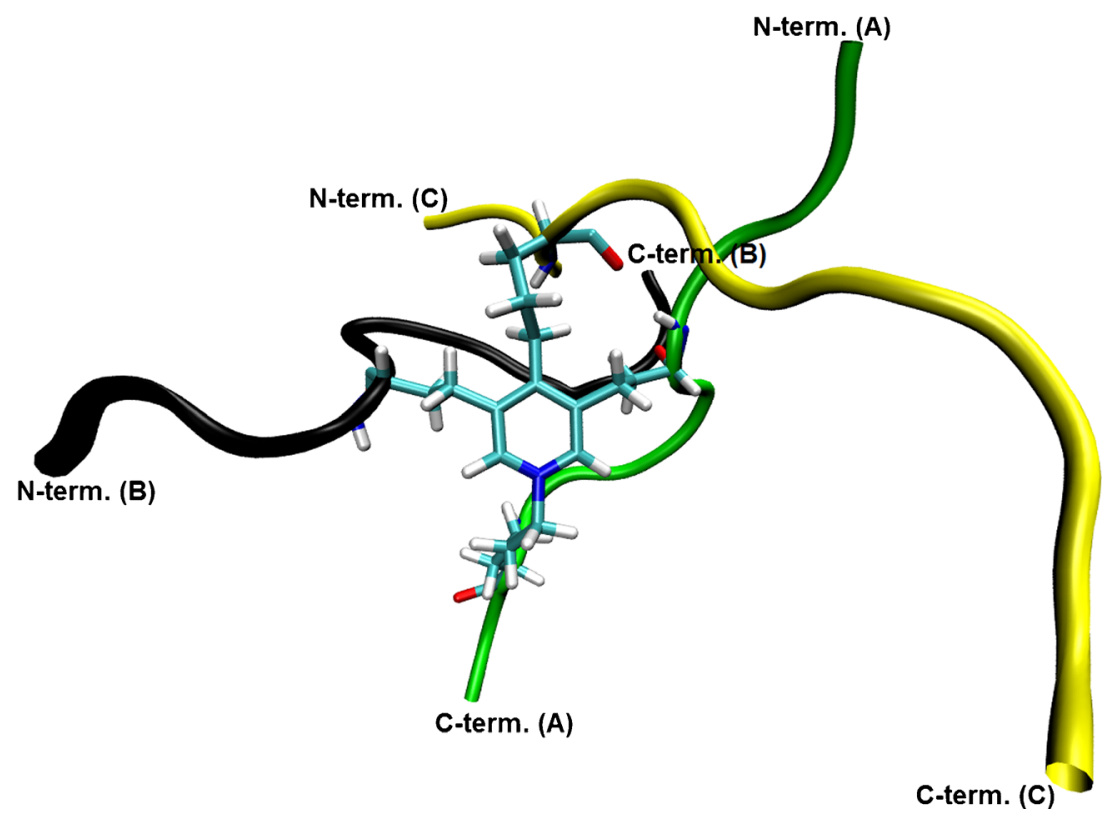

Figure 1. Conformation of a synthetic DES-/IDES-containing peptide formed by condensation reaction of three identical peptide monomers of the sequence Ac-AAAkAAAKAA. The residues involved in the cross-link formation are three allysines (one of each peptide) as well as one Lys from one of the peptides

from Merck (Darmstadt, Germany). HPLC-grade acetonitrile (VWR Prolabo, Leuven, Belgium), HPLC-grade methanol (VWR Prolabo), and doubly distilled water were used. All other chemicals were of analytical grade or higher purity.

\section{Isolation of Bovine Aortic Elastin}

Bovine aorta from one male animal was obtained from a local slaughterhouse. Prior to isolation, the aortic sample was cut into small pieces (lateral length of 5 to $10 \mathrm{~mm}$ ) and each piece was placed in a $1.5 \mathrm{~mL}$ reaction tube. Isolation of intact aortic elastin was carried out as described previously [8]. Purified elastin was allowed to dry at room temperature and was subsequently stored at $-26^{\circ} \mathrm{C}$ until analysis.

\section{Proteolysis and Fractionation of Bovine Aortic Elastin}

Elastin was dispersed in $50 \mathrm{mM}$ Tris $\mathrm{HCl}, \mathrm{pH} 8.5$, at a concentration of $5 \mathrm{mg} \mathrm{mL}^{-1}$. PE was added to a final enzyme-tosubstrate ratio of $1: 100(\mathrm{w} / \mathrm{w})$ and the samples were subsequently incubated at $37^{\circ} \mathrm{C}$ for $24 \mathrm{~h}$. The digestion was stopped by adding TFA to a final concentration of $0.5 \%(\mathrm{v} / \mathrm{v})$, and the samples were stored at $-26^{\circ} \mathrm{C}$ prior to further analysis. Fractionation of elastin digests was performed using an Agilent 1100 (Waldbronn, Germany) coupled to a Fraction Collector II (Waters, Manchester, UK) at a constant flow rate of $200 \mu \mathrm{L} \mathrm{min}{ }^{-1}$. For chromatographic separation, the injected sample was loaded onto a Reprosil-Pur 120 column (C18, $3 \mu \mathrm{m}, 2 \mathrm{~mm}$ i.d. $\times 150 \mathrm{~mm}$; Dr. Maisch GmbH, Ammerbuch-Entringen, Germany) and eluted using a solvent system of solvent $\mathrm{A}\left(0.1 \% \mathrm{FA}\right.$ in $\left.\mathrm{H}_{2} \mathrm{O}\right)$ and solvent $\mathrm{B}(0.1 \% \mathrm{FA}$ in $\left.\mathrm{ACN} / \mathrm{H}_{2} \mathrm{O} 80: 20(\mathrm{v} / \mathrm{v})\right)$ by applying a linear binary gradient:
$10 \%-40 \% \mathrm{~B}$ in $40 \mathrm{~min}$, to $90 \% \mathrm{~B}$ in the next $5 \mathrm{~min}$, followed by maintenance at $90 \% \mathrm{~B}$ for $10 \mathrm{~min}$, and then from $90 \%$ to $10 \% \mathrm{~B}$ in $5 \mathrm{~min}$. Fractions were collected every $30 \mathrm{~s}$.

\section{Proteolysis of Tropoelastin and nanoHPLC-nanoESI-QqTOF MS/MS Analysis}

Human TE (isoform 2) was dissolved in $50 \mathrm{mM}$ Tris $\mathrm{HCl}$, $\mathrm{pH} 8.5$, at a concentration of $1 \mathrm{mg} \mathrm{mL}^{-1}$. The digestion was carried out as described earlier. The PE digests of human TE were analyzed by nanoHPLC-nanoESI-QqTOF MS(/MS) experiments using an Ultimate 3000 nanoHPLC system (Thermo Fisher, Idstein, Germany) coupled to a Q-TOF-2 mass spectrometer (Waters, Manchester, UK) as described previously [29] with the exception of the CE, which was manually set between 60 and $180 \mathrm{eV}$ depending on mass and charge state. More precisely, the CE was set to values that allowed for a nearly complete dissociation of the peptide (i.e., where only immonium ions and other small internal fragment ions of $\mathrm{m} / \mathrm{z}$ values below 210 were observed).

\section{Derivatization of DES and IDES}

Fifty $\mu \mathrm{g}$ of DES and IDES were transferred to a $1.5 \mathrm{~mL}$ Eppendorf tube, respectively. Derivatization with propionic anhydride was carried out according to Boutin et al. [30]: $100 \mu \mathrm{L}$ of $0.1 \mathrm{~N} \mathrm{NH}_{4} \mathrm{HCO}_{3}$ and $100 \mu \mathrm{L}$ of a solution containing $23 \%(\mathrm{v} / \mathrm{v})$ propionic anhydride and $9 \%(\mathrm{v} / \mathrm{v}) \mathrm{NH}_{4} \mathrm{OH}$ were added to DES and IDES, respectively, and the samples were incubated at room temperature for $1 \mathrm{~h}$. Further derivatives of DES and IDES were prepared using amine 9-fluorenylmethyl chloroformate (FMOC; reacting at amine groups), butanol (reacting at the carboxylic acid groups), and propyl 
chloroformate ( $\mathrm{PrCl}$; reacting with both types) according to Uutela et al. [31]. All samples were evaporated to dryness using a centrifugal evaporator and then dissolved in $1 \mathrm{~mL}$ of $\mathrm{MeOH} / \mathrm{H}_{2} \mathrm{O}(80: 20, \mathrm{v} / \mathrm{v})$.

\section{In Vitro Cross-Linking of Short Elastin Peptides}

The elastin decapeptide Ac-AAAkAAAKAA was synthesized and cross-linked in vitro as described earlier [18]. Prior to static nanoESI-QqTOF MS/MS analysis, the supernatant containing linear as well as differently cross-linked peptides was evaporated to dryness and then dissolved in $\mathrm{MeOH} / \mathrm{H}_{2} \mathrm{O}(80: 20$, v/v).

\section{Static Tandem MS Experiments}

Static mass spectrometric analyses of DES/IDES, their derivatives, and the short cross-linked elastin peptide were carried out on Q-TOF-2 (Waters) and Orbitrap Velos Pro (Thermo Fisher Scientific, Bremen, Germany) mass spectrometers equipped with a nanoESI or ESI source, respectively. For nanoESI experiments, precoated glass emitters (PicoTips) from New Objective (Woburn, MA, USA) were used. Three microliters of each sample solution were loaded into the emitters using Microloader pipette tips (Eppendorf, Hamburg, Germany). The operating conditions of the MS were: capillary voltage, $1 \mathrm{kV}$; cone voltage, $35 \mathrm{~V}$; source temperature, $80^{\circ} \mathrm{C}$. Full scans were acquired in the positive ion mode over the $\mathrm{m} / \mathrm{z}$ range 50-2500 and precursor ions of interest were selected manually for CID tandem MS experiments. DES/IDES and their derivatives were observed singly and doubly charged with minor differences in their tandem mass spectra. However, for the sake of simplicity, we limit the results in this study to the singly charged species. CID experiments were carried out by varying CEs between 10 and $100 \mathrm{eV}$ increased in steps of $10 \mathrm{eV}$. For the corresponding linear and tetrafunctional peptides, the $\mathrm{CE}$ was raised until only immonium ions and small internal fragments with $m / z$ values below 210 were observed. Pseudo-MS ${ }^{3}$ spectra were acquired by applying an increased cone voltage (between 110 and $185 \mathrm{~V}$ ), thereby generating in-source CID fragments, which were further subjected to $\mathrm{MS}^{2}$ analysis. All product ion spectra were deconvoluted and de-isotoped by MassLynx 4.1 with the add-on MaxEnt 3 (Waters).

To facilitate elemental composition determination of product ions, selected species were subjected to higher-energy collisional dissociation (HCD) tandem MS on an LTQOrbitrap Velos Pro mass spectrometer. The capillary and spray voltages were set to $50 \mathrm{~V}$ and $3.5 \mathrm{kV}$, respectively. Tandem mass spectra from $\mathrm{m} / \mathrm{z} 50$ with a resolution of 100,000 (full width at half maximum) were acquired. The CEs were incrementally ramped up to $50 \mathrm{eV}$ for normal measurements and up to $161 \mathrm{eV}$ for measurements with enhanced fragmentation. Elemental compositions were calculated using the Waters tool Elemental Composition (v. 4.0) by considering the following elements and limits: $\mathrm{C}$ (0 to 30$) ; \mathrm{H}$ (0 to 100$) ; \mathrm{N}$ (0 to 10$) ; \mathrm{O}(0$ to 10$)$; $\mathrm{S}$ (0 to 10). Furthermore, an error tolerance of $3 \mathrm{ppm}$ was used as a constraint.

\section{Results and Discussion}

\section{Collision-Induced Dissociation of DES and IDES}

Owing to the fact that the pyridinium nitrogen in DES and IDES (Figure 2) has a fixed positive charge, no additional protonation is needed for ionization and, thus, the observed $\mathrm{m} / \mathrm{z}$ value for the singly charged precursor ion $[\mathrm{M}]^{+}$at $\mathrm{m} / \mathrm{z}$ 526.2871 is identical to the monoisotopic molecular mass of DES/IDES. Dissociation of the singly charged precursor ion at $40 \mathrm{eV}$ leads to a complex tandem mass spectrum in which DES and IDES show the same fragment ions that only differ in their relative intensities (Figure 2). Fragmentation is mainly characterized by the combined neutral losses of $\mathrm{NH}_{3}$ and $\mathrm{CO}$ (sum of $45.0214 \mathrm{u}$ ) from one of the four aliphatic chains and the dissociation of the $\mathrm{C}_{\varepsilon}-\mathrm{N}$ bond of the residual Lys chain at the pyridinium nitrogen [28]. The most abundant peaks in the fragment spectra are a result of the combination of these processes. Since amino acid groups present in DES/IDES are almost equivalent in their chemical environment, none is preferred over the others during fragmentation. This can be exemplified by the ion at $m / z 481$, which actually corresponds to four different fragments resulting from $\mathrm{CO}$ and $\mathrm{NH}_{3}$ loss of the alkyl chains at position 1, 3, 4, and 5 in DES and at positions $1,2,3$, and 5 in IDES, respectively. In general, the progressive loss of $\mathrm{CO}$ and $\mathrm{NH}_{3}$ leads to the following ion series: $\mathrm{m} / z 526$ $\left([\mathrm{DES} / \mathrm{IDES}]^{+}\right) \rightarrow 481\left(\left[\mathrm{DES} / \mathrm{IDES}-\mathrm{CO}-\mathrm{NH}_{3}\right]^{+}\right) \rightarrow 436$ $\left(\left[\mathrm{DES} / \mathrm{IDES}-2 \mathrm{CO}-2 \mathrm{NH}_{3}\right]^{+}\right) \rightarrow 391$ ([DES/IDES $-3 \mathrm{CO}-$ $\left.\left.3 \mathrm{NH}_{3}\right]^{+}\right) \rightarrow 346\left(\left[\mathrm{DES} / \mathrm{IDES}-4 \mathrm{CO}-4 \mathrm{NH}_{3}\right]^{+}\right)$. We propose that this combined loss of $\mathrm{CO}$ and $\mathrm{NH}_{3}$ is driven by the formation of a zwitterion of the corresponding functional amino acid groups, which at first leads to the loss of the former positively charged $\mathrm{NH}_{3}$. Consequently, the negatively charged oxygen forms an oxiranone through nucleophilic attack at the adjacent carbon that subsequently loses CO (shown in Scheme 1). The resulting chain still possesses a carbonyl function and the positive charge remains at the pyridinium nitrogen during the whole process. No other functional groups in the molecule are involved. This fragmentation hypothesis is supported by the fact that the described loss even occurs for a fragment at $\mathrm{m} / \mathrm{z} 391$, which possesses only one intact alkyl side chain as proven by pseudo-MS ${ }^{3}$ measurements (Figure 3a).

Another prominent ion series occurs at $\mathrm{m} / \mathrm{z}$ values $526 \rightarrow$ $397\left(\left[\mathrm{DES} / \mathrm{IDES}-\mathrm{C}_{6} \mathrm{H}_{11} \mathrm{NO}_{2}\right]^{+}\right) \rightarrow 352$ ([DES/IDES $\left.\left.\mathrm{C}_{6} \mathrm{H}_{11} \mathrm{NO}_{2}-\mathrm{CO}-\mathrm{NH}_{3}\right]^{+}\right) \rightarrow 307\left(\left[\mathrm{DES} / \mathrm{IDES}-\mathrm{C}_{6} \mathrm{H}_{11} \mathrm{NO}_{2}\right.\right.$ $\left.\left.-2 \mathrm{CO}-2 \mathrm{NH}_{3}\right]^{+}\right) \rightarrow 262\left(\left[\mathrm{DES} / \mathrm{IDES}-\mathrm{C}_{6} \mathrm{H}_{11} \mathrm{NO}_{2}-3 \mathrm{CO}-\right.\right.$ $\left.3 \mathrm{NH}_{3}\right]^{+}$) and results from the loss of the Lys residue at the pyridinium nitrogen in combination with $\mathrm{CO}$ and $\mathrm{NH}_{3}$ losses at each alkyl chain. If the charge is directed to the adjacent residual Lys chain lacking the terminal amino group because it is part of the pyridinium core, a fragment ion at $\mathrm{m} / \mathrm{z} 130 \mathrm{can}$ be observed, which also has been described to occur upon fragmentation of protonated Lys as protonated pipecolic acid. Further loss of $\mathrm{C}_{2} \mathrm{H}_{2} \mathrm{O}$ leads to the formation of an ion at $\mathrm{m} / \mathrm{z}$ 84 , which is well known as a prominent ion during dissociation of Lys-containing linear peptides [32]. A CE-dependent 

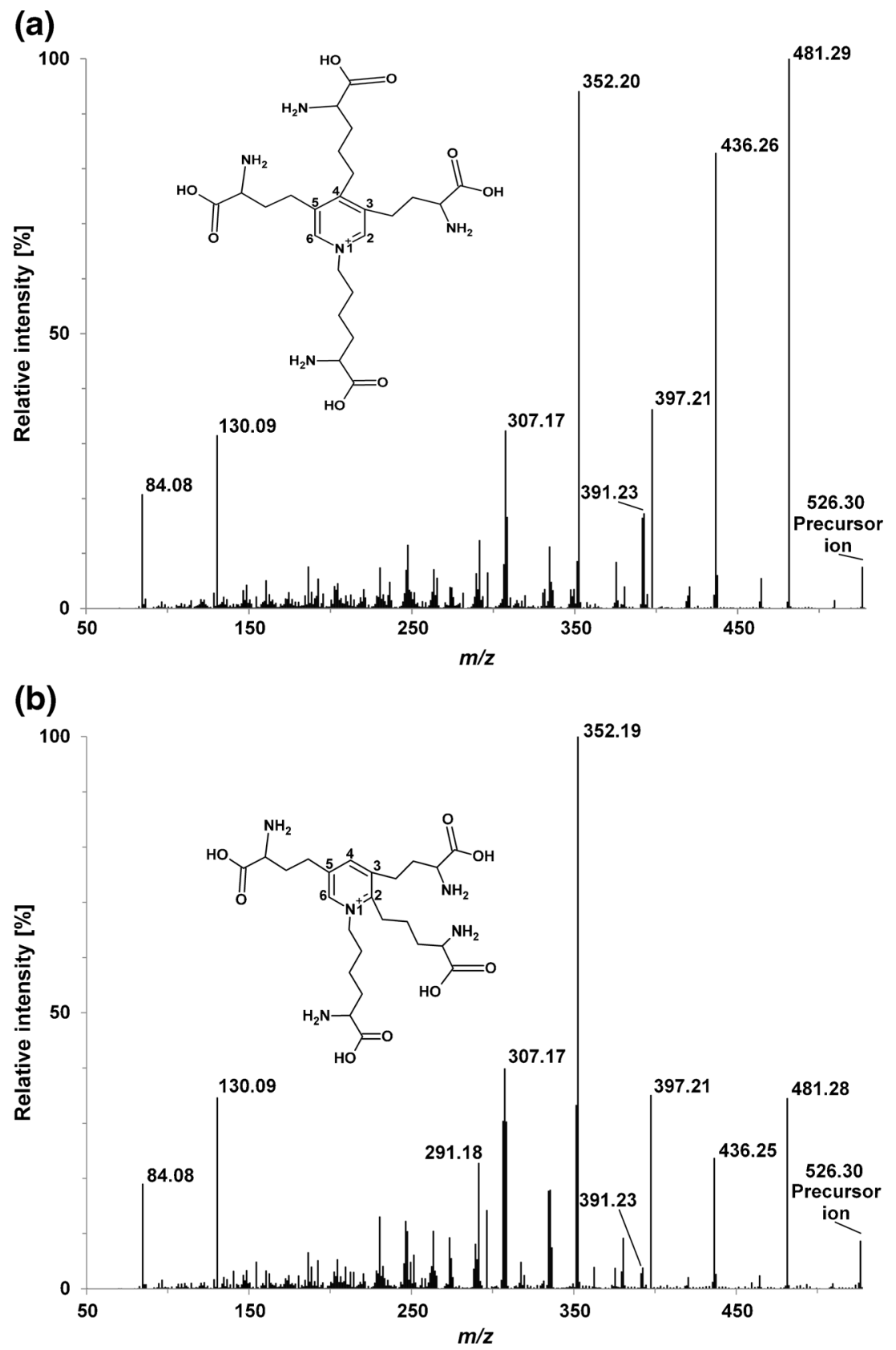

Figure 2. Chemical structures and positive MS/MS of (a) DES and (b) IDES acquired in CID mode at $40 \mathrm{eV}$ CE on a nanoESI-QqTOF instrument

analysis of the DES/IDES fragment ions is shown in Figure 4. It becomes evident that dissociation of IDES results in fragments predominantly characterized by the loss of the Lys side chain $(\mathrm{m} / \mathrm{z} 397,352$, and 307); whereas dissociation of DES is dominated by subsequent losses of $\mathrm{CO}$ and $\mathrm{NH}_{3}$ (corresponding fragment ions are $m / z 481,436,391$, and 346). In addition to the fragments displayed in Figure 4, several other fragments occur when the $\mathrm{CE}$ is further increased. These fragments represent further losses of small molecules (e.g., $\mathrm{H}_{2} \mathrm{O}$ and $\mathrm{CO}$ ). It is worth mentioning that dissociation of<smiles>[R]CCC(N)C(=O)OCPCCC([NH3+])C(=O)OCCC1OC1C(=O)OCCCC=O</smiles>

Scheme 1. Mechanism of the combined loss of $\mathrm{NH}_{3}$ and $\mathrm{CO}$ from one of the four attached aliphatic chains 

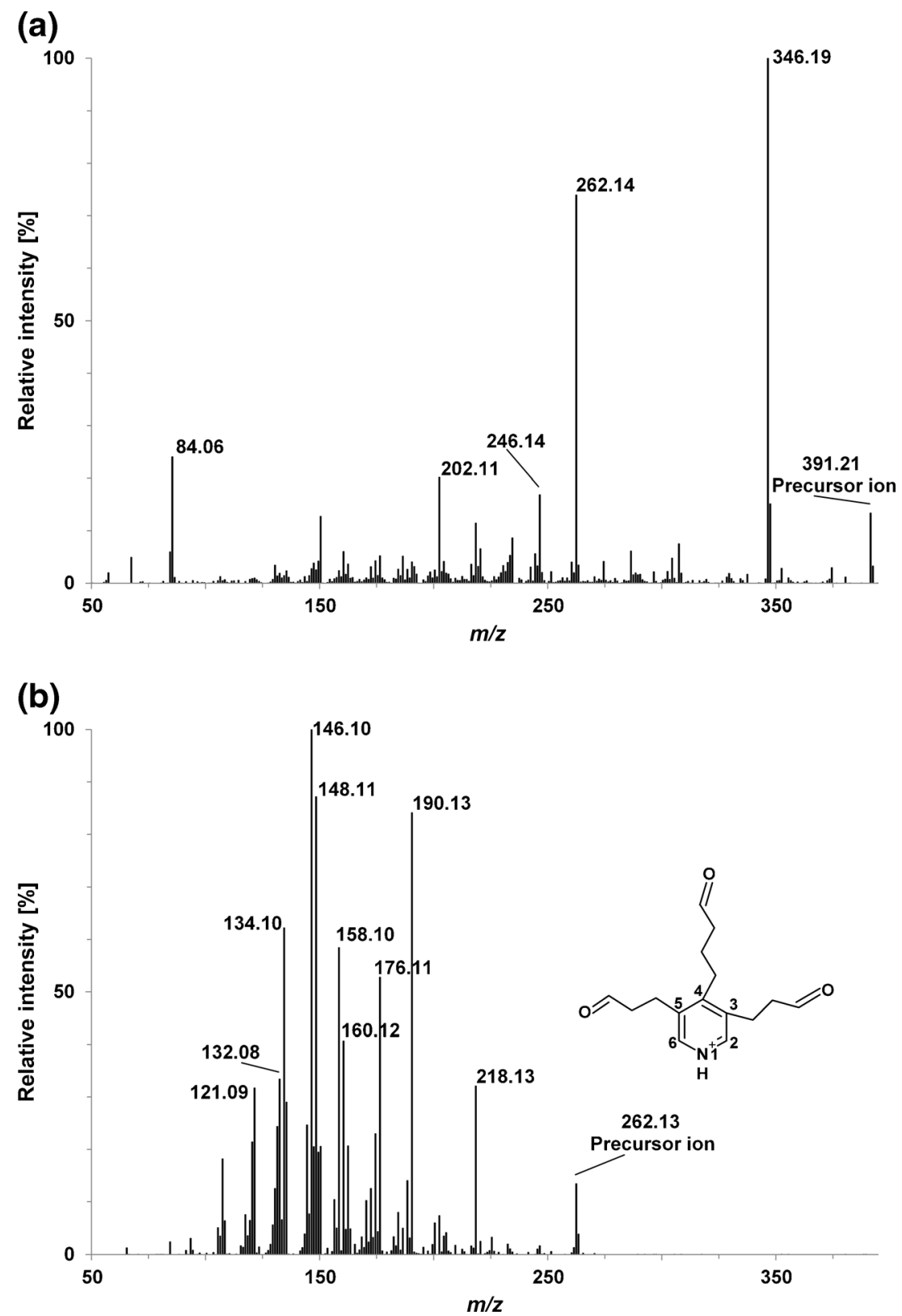

Figure 3. Pseudo-MS ${ }^{3} \mathrm{CID}$ spectra of DES product ions (a) $\mathrm{m} / \mathrm{z} 391$ and (b) $\mathrm{m} / \mathrm{z} 262$. The spectra were acquired in positive ion mode on a nanoESI-QqTOF instrument

DES and IDES at CEs above $60 \mathrm{eV}$ leads to the formation of an ion cluster resembling a Gaussian-like distribution, which occurs in the lower $\mathrm{m} / \mathrm{z}$ range. Elemental composition analyses of these product ions reveal molecular formulas of the type $\mathrm{C}_{\mathrm{x}} \mathrm{H}_{\mathrm{y}} \mathrm{N}(\mathrm{x}=9$ to $13, \mathrm{y}=7$ to 15$)$. CE-dependent formation of those ions is exemplified by $\mathrm{m} / \mathrm{z}$ values of 158,146 , and 132 in Figure 4. A detailed list of resulting product ions at elevated energies is shown in Table 1.

As mentioned above, the product ion at $\mathrm{m} / \mathrm{z} 262$ is formed by loss of all possible $\mathrm{CO}$ and $\mathrm{NH}_{3}$ groups in concert with the loss of the residual Lys side chain at the pyridinium nitrogen of DES/IDES. At $60 \mathrm{eV}$ it is the most abundant product ion. As shown by pseudo-MS ${ }^{3}$ data in Figure 3b, the cluster of product ions is also released from this fragment ion. A proposed dissociation pathway of $\mathrm{m} / \mathrm{z} 262$ is shown in Supplementary Figure S1. Overall, there is no evidence that the pyridinium core ring opens during CID of DES/IDES even at CEs above $100 \mathrm{eV}$. The generation of this series of fragment ions is likely to be driven by further charge-remote processes causing additional loss of oxygen atoms in combination with the loss of carbon and hydrogen atoms. Thus, product ions are formed that still contain the pyridinium moiety and attached alkyl chains. The latter differ in length and number of saturated bonds. This observation is consistent with findings of an earlier study of pyridinium-derivatized molecules [27]. Previous studies have underlined the stability of the pyridinium core against 

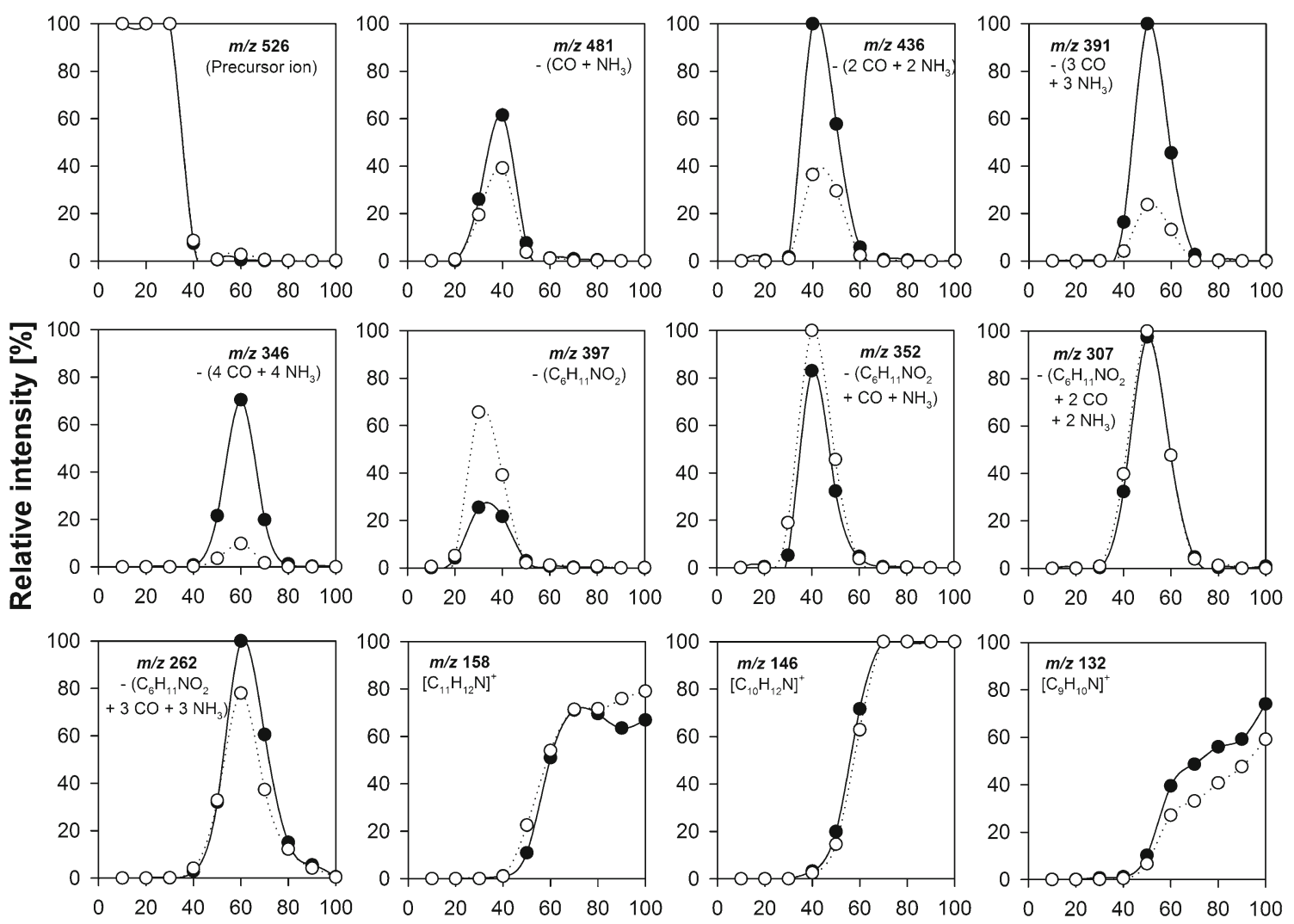

Collision energy $[\mathrm{eV}]$

Figure 4. Abundance of characteristic product ions of DES (filled circle) and IDES (circles) as a function of the applied CE. All fragment ion intensities are displayed relative to the base peak intensity at the respective CE

dissociation for several other pyridinium-derived compounds $[33,34]$. The stability of this core can be explained by its aromatic properties and the fixed charge at the nitrogen. As mentioned above, the product ion at $\mathrm{m} / \mathrm{z} 84$ is released at elevated energies but cannot serve as marker ion for DES-/ IDES-containing peptides as it is also produced during dissociation of Lys-containing linear peptides.
Interestingly, at $100 \mathrm{eV}$ the fragmentation patterns of DES and IDES are nearly identical even when taking into account the relative intensities of the fragment ions. Therefore, it is impossible to distinguish between them. Overall, the fragmentation behavior of DES/IDES is completely different from proteinogenic amino acids. When subjected to CID, common amino acids produce immonium ions of which some undergo

Table 1. Elemental Composition Analysis of Major DES and IDES Fragment Ions Observed at a CE of $100 \mathrm{eV}$

\begin{tabular}{lccc}
\hline$m / z(1+)$, Observed & $m / z(1+)$, Calculated & Mass error [ppm] & Elemental formula \\
\hline 188.1429 & 188.1434 & 2.66 & {$\left[\mathrm{C}_{13} \mathrm{H}_{18} \mathrm{~N}\right]^{+}$} \\
186.1276 & 186.1277 & 0.54 & {$\left[\mathrm{C}_{13} \mathrm{H}_{16} \mathrm{~N}\right]^{+}$} \\
184.1120 & 184.1121 & 0.54 & {$\left[\mathrm{C}_{13} \mathrm{H}_{14} \mathrm{~N}\right]^{+}$} \\
182.0959 & 182.0964 & 2.75 & {$\left[\mathrm{C}_{13} \mathrm{H}_{12} \mathrm{~N}\right]^{+}$} \\
174.1276 & 174.1277 & 0.57 & {$\left[\mathrm{C}_{12} \mathrm{H}_{16} \mathrm{~N}\right]^{+}$} \\
172.1118 & 172.1121 & 1.74 & {$\left[\mathrm{C}_{12} \mathrm{H}_{14} \mathrm{~N}\right]^{+}$} \\
170.0962 & 170.0964 & 1.18 & {$\left[\mathrm{C}_{12} \mathrm{H}_{12} \mathrm{~N}\right]^{+}$} \\
160.1118 & 160.1121 & 1.87 & {$\left[\mathrm{C}_{11} \mathrm{H}_{14} \mathrm{~N}\right]^{+}$} \\
158.0960 & 158.0964 & 2.53 & {$\left[\mathrm{C}_{11} \mathrm{H}_{12} \mathrm{~N}\right]^{+}$} \\
156.0806 & 156.0808 & 1.28 & {$\left[\mathrm{C}_{11} \mathrm{H}_{10} \mathrm{~N}\right]^{+}$} \\
146.0962 & 146.0964 & 1.37 & {$\left[\mathrm{C}_{10} \mathrm{H}_{12} \mathrm{~N}\right]^{+}$} \\
144.0805 & 144.0808 & 2.08 & {$\left[\mathrm{C}_{10} \mathrm{H}_{10} \mathrm{~N}\right]^{+}$} \\
134.0962 & 134.0964 & 1.49 & {$\left[\mathrm{C}_{9} \mathrm{H}_{12} \mathrm{~N}\right]^{+}$} \\
132.0805 & 132.0808 & 2.27 & {$\left[\mathrm{C}_{9} \mathrm{H}_{10} \mathrm{~N}\right]^{+}$} \\
130.0649 & 130.0651 & 1.54 & {$\left[\mathrm{C}_{9} \mathrm{H}_{8} \mathrm{~N}\right]^{+}$} \\
\hline
\end{tabular}


further fragmentation [22, 35]. For DES/IDES, however, this is hindered by the combined progressive loss of $\mathrm{CO}$ and $\mathrm{NH}_{3}$.

\section{Dissociation of Derivatives of DES and IDES}

Other DES-/IDES-containing molecules were required to investigate whether the above-mentioned product ions are also formed when the four amino and four carboxyl termini are modified. Therefore, different derivatization reactions were used for modifying DES/IDES by introducing uncharged functional groups at the termini. These derivatives also carry a permanent positive charge at the pyridinium nitrogen. The singly and doubly charged precursor ions of all derivatives were subjected to CE-dependent fragmentation. Their structural formulas are displayed in Scheme 2. Overall, collisioninduced dissociation of FMOC-DES/FMOC-IDES led to product ions that had gradually lost the FMOC groups yielding free DES/IDES product ions [31]. These subsequently dissociated further as described earlier. When a charge was transferred to the FMOC group during dissociation of the doubly charged precursor ion, prominent product ions of FMOC at $m / z 178$ and 179 were observed [31]. Fragmentation processes of $\mathrm{PrCl}-$ DES/PrCl-IDES, Pr-DES/Pr-IDES and butanol-DES/butanolIDES are characterized by neutral losses of small molecules at the modified amino acid groups. For instance, dissociation of butanol-DES/butanol-IDES involves the loss of 1-butene, CO, and $\mathrm{NH}_{3}$. The dissociation of the $\mathrm{C}_{\varepsilon}-\mathrm{N}$ bond at the pyridinium core was also observed for these three compounds. Since most product ions originated from several concurrent dissociation reactions, fragmentation yielded complex mass spectra even at low CEs in the range of 30 to $40 \mathrm{eV}$. Dissociation of the pyridinium ring was not observed, and further increase of the $\mathrm{CE}$ up to $110 \mathrm{eV}$ led to the same characteristic ion cluster as seen for free DES/IDES (Figure 5a and b). This is due to the fact that the dissociation channels of both DES/IDES and their derivatives are virtually identical. However, dissociation of the latter involves cleavages close to the derivatized amino acid groups followed by dissociations of the attached alkyl chains. As an example, the fragment spectra of Pr-DES/Pr-IDES at $110 \mathrm{eV}$ are shown in Figure 5c and d, respectively.

\section{Dissociation of In Vitro Cross-Linked Peptides}

The fact that DES/IDES as well as their derivatives show the same product ions at elevated CEs raised the question of whether fragmented peptides containing DES/IDES show this characteristic distribution of fragment ions. To answer this question, the fragmentation behavior of a synthetic in vitro cross-linked DES-/IDES-containing peptide (Figure 1) at $\mathrm{m} / \mathrm{z}$ 1298 (2+), described earlier [18], was investigated. Stepwise increase of the $\mathrm{CE}$ up to $180 \mathrm{eV}$ yielded a fragmentation pattern with the typical DES/IDES product ions of $\mathrm{C}_{\mathrm{x}} \mathrm{H}_{\mathrm{y}} \mathrm{N}$ as well as the $\mathrm{a} 1$ and $\mathrm{b} 1$ ions of Ac-Ala and a product ion at $\mathrm{m} / \mathrm{z} 84$, which is released from both DES/IDES and Lys (Figure 5e). The latter is of high intensity in the spectrum as the peptide has two additional Lys residues. It is worth mentioning that no peaks of the described product ion cluster were observed when applying CEs that are commonly used for the fragmentation of peptides (see Supplementary Figure S2).

\section{Dissociation of Linear Peptides}

In order to exclude the possibility that linear peptides present in elastin-containing digests also show the formation of product ions similar or equal in mass to those found for DES/IDES and derivatives, a set of 314 pre-identified peptides generated upon digestion of human TE by PE was analyzed by LC-MS/MS using data-dependent acquisition (DDA). For this purpose, elevated CEs as described earlier were stored in lookup tables. The energies were then selected automatically according to the charge state and mass value of the respective precursor ion during the measurements. Fragmentation of linear peptides<smiles>[R2]NC(CCCc1c(CCC(N[R2])C(=O)O[R2])c[n+](CCCC(N[R2])C(=O)O[R6])cc1CCC(N[R2])C(=O)O[R6])C(=O)O[R6]</smiles>

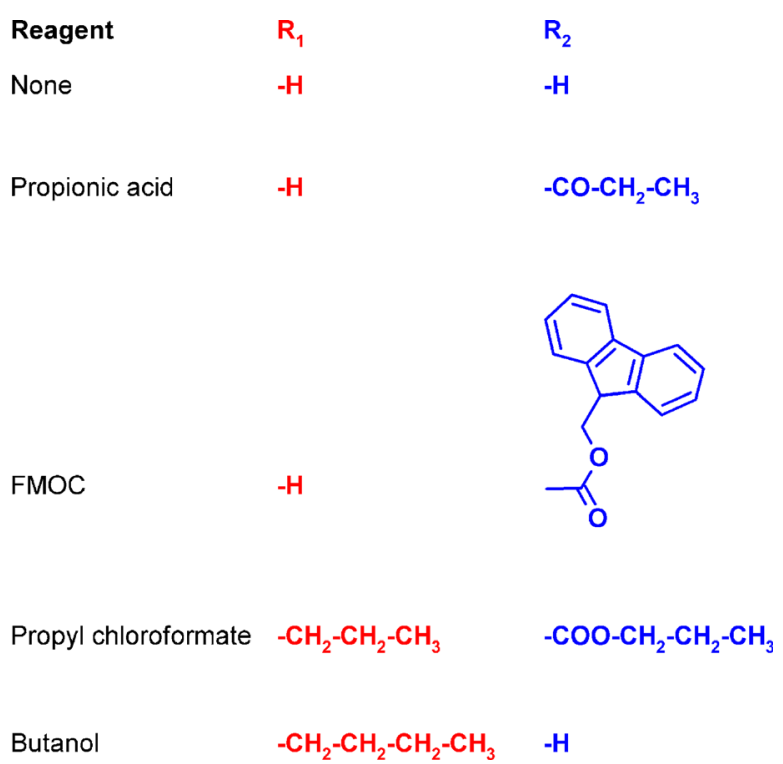

Scheme 2. Chemical structures of the four derivatives, depicted for DES 
(a)

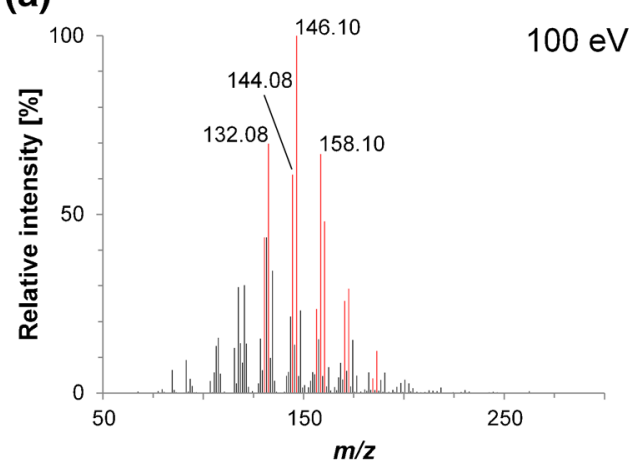

(c)

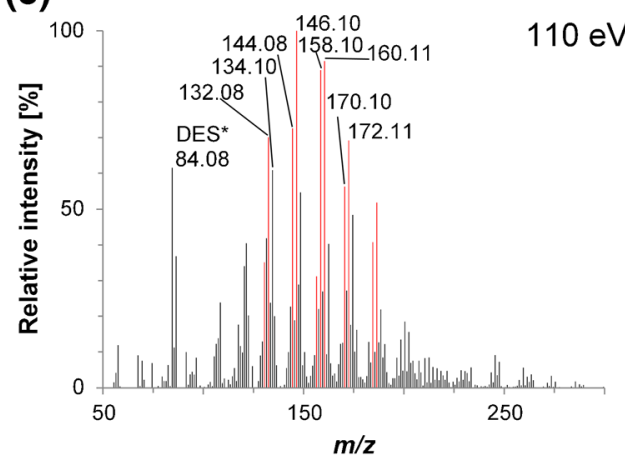

(e)

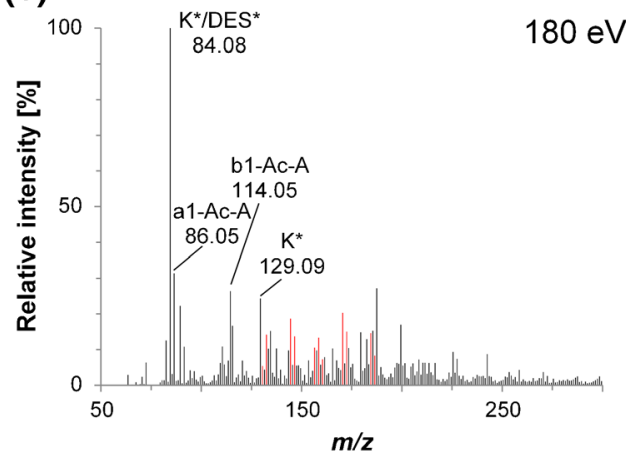

(g)

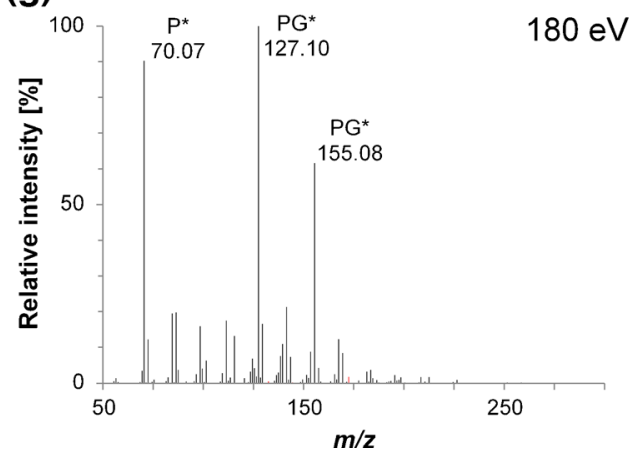

(b)

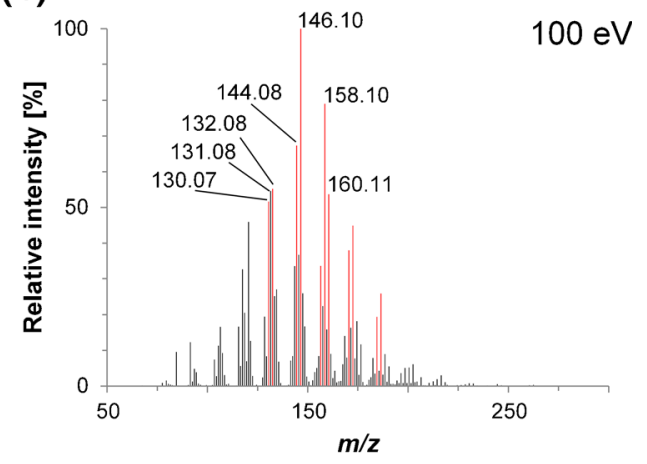

(d)

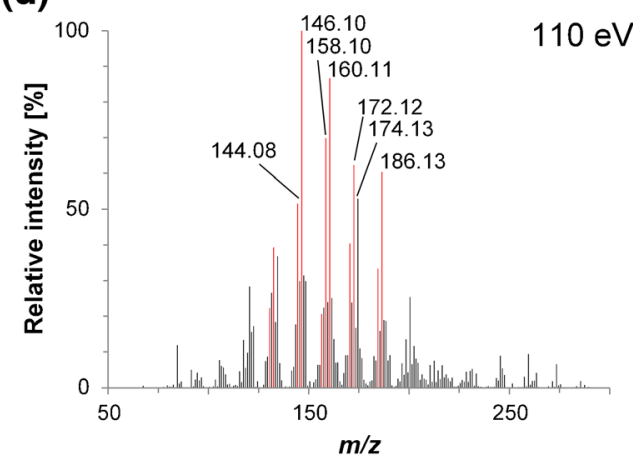

(f)

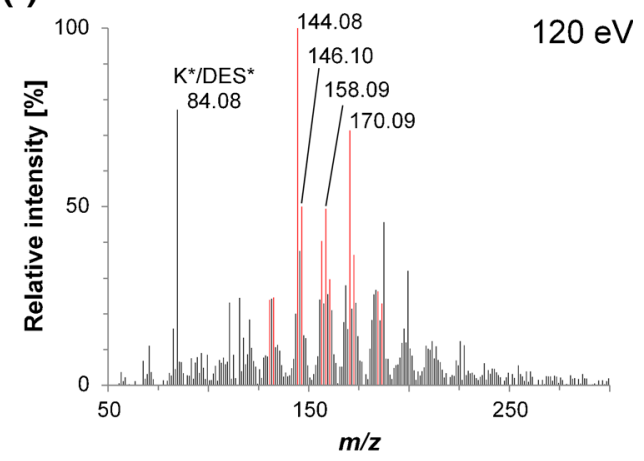

(h)

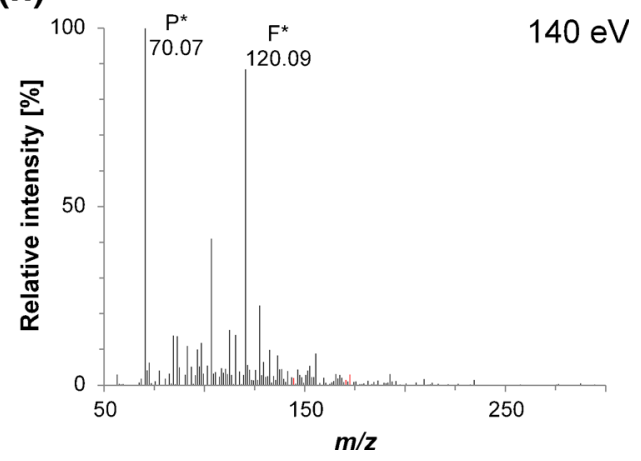

Figure 5. CID spectra of (a) DES, (b) IDES, (c) Pr-DES, (d) Pr-IDES, (e) an in vitro cross-linked DES-/IDES-containing elastin peptide formed by reaction of three identical peptide monomers with the sequence Ac-AAAkAAAKAA (2595.49 u; amino acids involved in cross-linking highlighted in bold), (f) a DES-containing peptide from a digest of bovine aortic elastin $(1449.61 \mathrm{u})$, (g) the linear peptide GVLPGVGGAGVPGVPGAIPGIGGIAGVGTPA (2521.37 u), and (h) the linear peptide AARPGFGLSPIFPGGA (1514.75 u). The two linear peptides were identified in PE digests of TE. Eleven abundant fragment ions characteristic of DES-/IDES-containing species are highlighted in red. Internal fragments, immonium ions, and related ions as well as other are labeled with an asterisk 
induced by elevated CEs leads to the formation of a few low mass product ions. Two typical fragment spectra observed for linear peptides are shown in Figure 5g and h. Immonium ions (e.g., of Pro or Phe) are abundant, whereas other fragments are rare and of low intensity. Overall, neither masses equal to those of typical fragments of DES/IDES and their derivatized forms nor the characteristic fragment cluster resembling a Gaussian-like distribution were observed in fragment spectra of any linear peptides.

\section{Development of an Algorithm for the Rapid Identi- fication of Tetrafunctionally Cross-Linked Peptides}

Based on the fact that DES-/IDES-containing peptide species show characteristic fragment ions at elevated CEs that are unlikely to be generated during fragmentation of linear peptides, a software tool was developed, which allows differentiation of linear and tetrafunctionally cross-linked elastin peptides in LC-MS data of protein digests. For this purpose, a common DDA method with elevated CEs can be applied. The software, which is available upon request, searches de-isotoped peak lists in the DTA, PKL, or MGF format for an array of 11 abundant marker ions $[130.07 ; 132.08 ; 144.08 ; 146.10$; $156.08 ; 158.10 ; 160.11 ; 170.10 ; 172.11 ; 182.10 ; 186.13$ ] whose individual intensities are compared to the base peak intensity of all marker ions by applying a threshold of 5\%. The number of marker peaks $n$ that meets this criterion is used to calculate a score $s$ that can assume values between 0 and 1 based on the following equation: $\mathrm{s}=1.00408677 /(1+\operatorname{Exp}[-$ 1.1(n-6)]). This sigmoid function turned out to be a suitable model for the differentiation of linear and DES-/IDES-containing peptides. The relation between matched product ions and score is illustrated in the insert in Figure 6. Only the appearance of a high number of marker fragments results in a high score (close to 1), whereas for instance noisy spectra that by chance possess a low number of marker fragments will receive a low score. The set of fragment spectra derived from 314 linear peptides was used as negative control/training set and was taken into consideration for the development of the algorithm. Calculated scores based on $\mathrm{MS}^{2}$ data of the discussed compounds are shown in Figure 6.

\section{Identification of Cross-Linked Peptides Containing DES/IDES in Digests of Bovine Aortic Elastin}

The developed approach was used to identify cross-linked peptides containing DES or IDES of mature elastin that was isolated from bovine aortic tissue prior to digestion with PE. Nine peptides with high scores and masses ranging from 1250 to $1900 \mathrm{Da}$ were found in early fractions of the chromatographic separation. The rapid elution of such cross-linked peptides is caused by their low ability to interact with the $\mathrm{C} 18$ material because of their high polarity. All these peptides showed the characteristic cluster of low mass product ions upon CID-MS/ MS using elevated CEs. The tandem mass spectrum of a doubly charged species $(\mathrm{m} / \mathrm{z} 725)$ with typical marker fragments highlighted is shown in Figure 5f. To illustrate the similarity between different DES species, tandem mass spectra of the low mass range of DES, Pr-DES, and this peptide are displayed in Supplementary Figure S3. CID of linear peptides proceeds through proton-mediated fragmentation processes, whereas in the case of permanently charged species such as DES-/IDES-containing peptides, fragmentation relies on

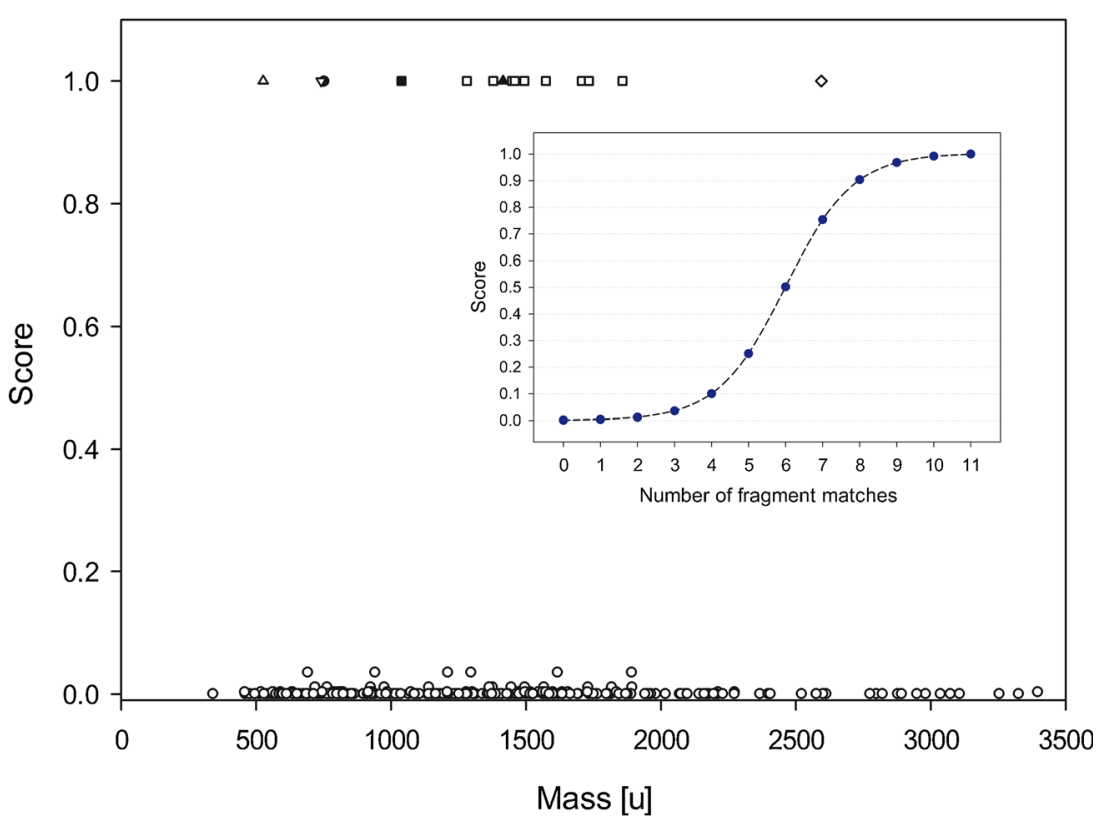

Figure 6. Scores obtained for DES/IDES (upright triangle), Pr-DES/Pr-IDES (750.39 u; reversed triangle), FMOC-DES/FMOC-IDES (1414.56 u; filled upright triangle), butanol-DES/butanol-IDES (750.54 u; filled circle), PrCl-DES/PrCl-IDES (1038.62 u; filled square), bovine DES-/IDES-containing elastin peptides (squares), and an in-vitro cross-linked elastin peptide (rotated square). A set of 314 non-cross-linked elastin peptides (circles) was used as control. All data were acquired on a QqTOF instrument. The insert shows the relation between matched product ions and resultant score based on a sigmoid function 
charge-remote processes [27]. The species found in these samples were predominantly doubly charged - with one fixed charge - and thus, fragmentation of these molecular ions proceeds through both processes. However, the pyridinium moiety persists in either case and the described marker fragments are observable at elevated CEs. Since the algorithm takes 11 marker fragment ions into account, the detection of tetrafunctionally cross-linked peptides is also possible on instruments with lower resolution and mass accuracy. To further confirm the presence of DES/IDES in these peptides, appropriate fractions were totally hydrolyzed, derivatized, and then subjected to tandem MS as described in a previous work [36]. The release of DES/ IDES was thus proven for all fractions in which DES-/IDEScontaining peptides were detected. Further investigation of these peptides is necessary to gain insight into the involved cross-linking domains.

\section{Conclusions}

DES and IDES are important marker molecules as their release indicates the irreversible breakdown of the elastic fiber network in severe diseases. Although these crosslinks are excreted not only as free amino acids but also as integral parts of peptides, virtually nothing has been reported with regard to their identity. However, such peptides would provide important insights about the cleavages that take place in elastin and, furthermore, promote the elucidation of elastin's cross-linking pattern, which, despite all efforts, is still unknown.

In this study, we investigated the dissociation behavior of DES and IDES, several of their derivatives, as well as DES-/ IDES-containing peptides by CID-MS/MS. It was found that a cluster of fragments is generated when applying elevated CEs. This cluster is characteristic for pyridinium-derived compounds and can, hence, serve as a fingerprint in DDA or precursor ion scanning experiments. It may be useful as a straightforward assay for the discovery of DES-/IDES-containing peptides in proteolytic digests of body fluids. This was proven by the detection of such peptides in digests of purified mature elastin derived from bovine aorta. The approach represents a promising step towards the elucidation of the yet unknown cross-linking pattern of mature elastin, and future work will focus on further investigation and exact sequence identification of such DES- and IDES-containing peptides in human tissues.

\section{Acknowledgments}

The authors are grateful to Dr. Alex G. Harrison for his valuable comments and suggestions. The work was financially supported by the German Research Foundation (DFG) grant HE 6190/1-2 (to A.H.) and by the European Regional Development Fund of the European Commission (to C.U.S.). The authors thank the anonymous reviewers for their valuable comments and suggestions.

\section{References}

1. Vrhovski, B., Weiss, A.S.: Biochemistry of tropoelastin. Eur. J. Biochem. 258, 1-18 (1998)

2. Mecham, R.P., Foster, J.A.: A structural model for desmosine cross-linked peptides. Biochem. J. 173, 617-625 (1978)

3. Akagawa, M., Suyama, K.: Mechanism of formation of elastin crosslinks. Connect. Tissue Res. 41, 131-141 (2000)

4. Bedell-Hogan, D., Trackman, P., Abrams, W., Rosenbloom, J., Kagan, H.: Oxidation, cross-linking, and insolubilization of recombinant tropoelastin by purified lysyl oxidase. J. Biol. Chem. 268, 10345-10350 (1993)

5. Mithieux, S.M., Weiss, A.S.: Elastin. Adv. Protein Chem. 70, 437-461 (2005)

6. Rucker, R.B., Murray, J.: Cross-linking amino acids in collagen and elastin. Am. J. Clin. Nutr. 31, 1221-1236 (1978)

7. Kozel, B.A., Wachi, H., Davis, E.C., Mecham, R.P.: Domains in tropoelastin that mediate elastin deposition in vitro and in vivo. J. Biol. Chem. 278, 18491-18498 (2003)

8. Schmelzer, C.E.H., Jung, M.C., Wohlrab, J., Neubert, R.H.H., Heinz, A.: Does human leukocyte elastase degrade intact skin elastin? FEBS J. 279, 4191-4200 (2012)

9. Wagenseil, J.E., Mecham, R.P.: Elastin in large artery stiffness and hypertension. J. Cardiovasc. Transl. Res. 5, 264-273 (2012)

10. Luisetti, M., Ma, S., Iadarola, P., Stone, P.J., Viglio, S., Casado, B., Lin, Y.Y., Snider, G.L., Turino, G.M.: Desmosine as a biomarker of elastin degradation in COPD: current status and future directions. Eur. Respir. J. 32, 1146-1157 (2008)

11. Campa, J.S., Greenhalgh, R.M., Powell, J.T.: Elastin degradation in abdominal aortic aneurysms. Atherosclerosis 65, 13-21 (1987)

12. O'Brien, J.P., Regan, W.: A study of elastic tissue and actinic radiation in "aging," temporal arteritis, polymyalgia rheumatica, and atherosclerosis. The actinic storm in the modern world. J. Am. Acad. Dermatol. 24, 765776 (1991)

13. Ma, S., Turino, G.M., Lin, Y.Y.: Quantitation of desmosine and isodesmosine in urine, plasma, and sputum by LC-MS/MS as biomarkers for elastin degradation. J. Chromatogr. B Analyt. Technol. Biomed. Life Sci. 879, 1893-1898 (2011)

14. Viglio, S., Annovazzi, L., Luisetti, M., Stolk, J., Casado, B., Iadarola, P.: Progress in the methodological strategies for the detection in real samples of desmosine and isodesmosine, two biological markers of elastin degradation. J. Sep. Sci. 30, 202-213 (2007)

15. Starcher, B., Sauter, E., Ho, C.: Elastin turnover in malignant solid tumors. Connect. Tissue Res. 54, 313-318 (2013)

16. Viglio, S., Stolk, J., Luisetti, M., Ferrari, F., Piccinini, P., Iadarola, P.: From micellar electrokinetic chromatography to liquid chromatography-mass spectrometry: revisiting the way of analyzing human fluids for the search of desmosines, putative biomarkers of chronic obstructive pulmonary disease. Electrophoresis 35, 109-118 (2014)

17. Mayne, S.L., Patterton, H.G.: Bioinformatics tools for the structural elucidation of multi-subunit protein complexes by mass spectrometric analysis of protein-protein cross-links. Brief. Bioinform. 12, 660-671 (2011)

18. Heinz, A., Ruttkies, C.K.H., Jahreis, G., Schräder, C.U., Wichapong, K., Sippl, W., Keeley, F.W., Neubert, R.H.H., Schmelzer, C.E.H.: In vitro cross-linking of elastin peptides and molecular characterization of the resultant biomaterials. Biochim. Biophys. Acta 1830, 2994-3004 (2013)

19. Schmelzer, C.E.H., Getie, M., Neubert, R.H.H.: Mass spectrometric characterization of human skin elastin peptides produced by proteolytic digestion with pepsin and thermitase. J. Chromatogr. A 1083, 120-126 (2005)

20. Wells, J.M., McLuckey, S.A.: Collision-induced dissociation (CID) of peptides and proteins. Methods Enzymol. 402, 148-185 (2005)

21. Ambihapathy, K., Yalcin, T., Leung, H.W., Harrison, A.G.: Pathways to immonium ions in the fragmentation of protonated peptides. J. Mass Spectrom. 32, 209-215 (1997)

22. Falick, A.M., Hines, W.M., Medzihradszky, K.F., Baldwin, M.A., Gibson, B.W.: Low-mass ions produced from peptides by high-energy collisioninduced dissociation in tandem mass-spectrometry. J. Am. Soc. Mass Spectrom. 4, 882-893 (1993)

23. Papayannopoulos, I.A.: The Interpretation of collision-induced dissociation tandem mass-spectra of peptides. Mass Spectrom. Rev. 14, 49-73 (1995)

24. Hohmann, L.J., Eng, J.K., Gemmill, A., Klimek, J., Vitek, O., Reid, G.E., Martin, D.B.: Quantification of the compositional information provided by immonium ions on a quadrupole-time-of-flight mass spectrometer. Anal. Chem. 80, 5596-5606 (2008) 
25. Hung, C.W., Schlosser, A., Wei, J.H., Lehmann, W.D.: Collision-induced reporter fragmentations for identification of covalently modified peptides. Anal. Bioanal. Chem. 389, 1003-1016 (2007)

26. Todorovski, T., Fedorova, M., Hoffmann, R.: Identification of isomeric 5hydroxytryptophan- and oxindolylalanine-containing peptides by mass spectrometry. J. Mass Spectrom. 47, 453-459 (2012)

27. Baker, A.G., Wiesler, D., Novotny, M.V.: Tandem mass spectrometry of model peptides modified with trans-2-hexenal, a product of lipid peroxidation. J. Am. Soc. Mass Spectrom. 10, 613-624 (1999)

28. Ongay, S., Hermans, J., Bruins, A.P., Nieuwendijk, A.M., Overkleeft, H., Bischoff, R.: Electron transfer and collision induced dissociation of nonderivatized and derivatized desmosine and isodesmosine. J. Am. Soc. Mass Spectrom. 24, 83-91 (2012)

29. Heinz, A., Jung, M.C., Jahreis, G., Rusciani, A., Duca, L., Debelle, L., Weiss, A.S., Neubert, R.H.H., Schmelzer, C.E.H.: The action of neutrophil serine proteases on elastin and its precursor. Biochimie 94, 192-202 (2012)

30. Boutin, M., Berthelette, C., Gervais, F.G., Scholand, M.B., Hoidal, J., Leppert, M.F., Bateman, K.P., Thibault, P.: High-sensitivity nanoLC-MS/ MS analysis of urinary desmosine and isodesmosine. Anal. Chem. 81, 1881-1887 (2009)
31. Uutela, P., Ketola, R.A., Piepponen, P., Kostiainen, R.: Comparison of different amino acid derivatives and analysis of rat brain microdialysates by liquid chromatography tandem mass spectrometry. Anal. Chim. Acta 633, 223-231 (2009)

32. Yalcin, T., Harrison, A.G.: Ion chemistry of protonated lysine derivatives. J. Mass Spectrom. 31, 1237-1243 (1996)

33. Denekamp, C., Tenetov, E., Horev, Y.: Homolytic cleavages in pyridinium ions, an excited state process. J. Am. Soc. Mass Spectrom. 14, 790-801 (2003)

34. Katritzky, A.R., Watson, C.H., Degaszafran, Z., Eyler, J.R.: Collisionally Activated dissociation of some pyridinium cations - novel fragmentation pathways. Org. Mass Spectrom. 24, 1017-1021 (1989)

35. Rogalewicz, F., Hoppilliard, Y., Ohanessian, G.: Fragmentation mechanisms of alpha-amino acids protonated under electrospray ionization: a collisional activation and ab initio theoretical study. Int. J. Mass Spectrom. 195, 565-590 (2000)

36. McGrath, A.P., Mithieux, S.M., Collyer, C.A., Bakhuis, J.G., van den Berg, M., Sein, A., Heinz, A., Schmelzer, C., Weiss, A.S., Guss, J.M.: Structure and activity of Aspergillus nidulans copper amine oxidase. Biochemistry 50, 5718-5730 (2011) 